\title{
Relating the large-scale structure of time series and visibility networks
}

\author{
Miguel A. Rodríguez \\ Instituto de Física de Cantabria (IFCA), CSIC-UNICAN, E-39005 Santander, Spain
}

(Received 21 March 2017; published 30 June 2017)

\begin{abstract}
The structure of time series is usually characterized by means of correlations. A new proposal based on visibility networks has been considered recently. Visibility networks are complex networks mapped from surfaces or time series using visibility properties. The structures of time series and visibility networks are closely related, as shown by means of fractional time series in recent works. In these works, a simple relationship between the Hurst exponent $H$ of fractional time series and the exponent of the distribution of edges $\gamma$ of the corresponding visibility network, which exhibits a power law, is shown. To check and generalize these results, in this paper we delve into this idea of connected structures by defining both structures more properly. In addition to the exponents used before, $H$ and $\gamma$, which take into account local properties, we consider two more exponents that, as we will show, characterize global properties. These are the exponent $\alpha$ for time series, which gives the scaling of the variance with the size as $\operatorname{var} \sim T^{2 \alpha}$, and the exponent $\kappa$ of their corresponding network, which gives the scaling of the averaged maximum of the number of edges, $\left\langle k_{M}\right\rangle \sim N^{\kappa}$. With this representation, a more precise connection between the structures of general time series and their associated visibility network is achieved. Similarities and differences are more clearly established, and new scaling forms of complex networks appear in agreement with their respective classes of time series.
\end{abstract}

DOI: 10.1103/PhysRevE.95.062309

\section{INTRODUCTION}

Connecting points of a discretized hypersurface that are mutually visible, one gets a network where the points are nodes and the links are edges. When the surface is rough enough, the corresponding network becomes complex. Then, it can be expected that both objects are closely related. This mapping was first used in the characterization of time series dynamics. A graphical representation of time series as one-dimensional (1D) discretized surfaces allows for the interpretation of dynamics events as topological facts [1]. Continuing with this suggestive idea, one can assume that this mapping can be interesting from two points of view. On the one hand, it can be a proper nonparametric method of analysis of some aspects of time series, while on the other hand it can be a simple method with which to build correlated networks with determined properties. Although only the first part of this proposition has been exploited [2-4], one can expect additional interaction between both subjects. Obviously, deeper knowledge of the relation between them is necessary. The objective of this paper is to obtain such knowledge.

Recently, the structure of time series and visibility networks was connected in the simple case of series generated from fractional Brownian (fB) processes [2,5]. In these works, a simple relationship was shown between the Hurst exponent $H$ of fractional time series and the exponent of the probability density of nodes $\gamma$ of the corresponding visibility network, which exhibits a power law. It reads $\gamma=2-3 H$ for series generated from fractional Brownian motion $(\mathrm{fBm})$ processes, and $\gamma=5-2 H$ for those generated from fractional Brownian noise $(\mathrm{fBn})$. Although these values are not definitive, this is a good example of the close relation between the geometrical objects, in this case a time series taken as a 1D solid surface, and its corresponding visibility network, i.e., the network obtained connecting visible points on this surface. The next step is of course to extend and check these results to more general types of series. For this aim, a more complete description of both objects is necessary. A standard way of describing the structure of a time series is by means of spectral properties or correlations. In a recent paper [6], it was shown that a complete time series analysis based on spectral properties requires knowledge of two types of behaviors, of local and global character, observed in changes of scale. Hence, there will be series with single or double spectral scaling. Series generated from $\mathrm{fB}$ processes are typically single-scaled, so only one exponent is necessary to characterize their structure, for instance the Hurst exponent $H$. In general, single scaling is observed in series generated from additive and linear processes. Those generated from multiplicative processes exhibit double scaling, and a description with at least two exponents has to be used [6].

On the other hand, in order to characterize more properly the structure of complex networks, besides the exponent given by the power of the degree distribution $\gamma$ [7], it is necessary to take into account another distinct property [8]. In the case of growing networks, a good choice is the scaling of the maximum degree with the size of the network, $\left\langle k_{M}\right\rangle \sim N^{\kappa}$, which produces a new exponent $\kappa$ [9]. With these representations in mind, we can see in the paper that a more precise connection between the structures of general time series and their associated visibility network is achieved. In Sec. II, a brief description of the method used for characterizing time series is presented. The method gives an ordering in classes using two exponents of local and global character. Five classes are selected to represent typical kinds of time series. Numerical methods to obtain synthetic samples of each class are outlined. Section III explains how to deal with the large-scale structure of complex networks by using two exponents with the same local and global character as the ones used in the previous section. Section IV introduces visibility networks and their properties. Section V presents a numerical analysis of the large-scale structure of visibility networks mapped from the five classes of time series introduced in Sec. II. Finally, Sec. VI concludes by emphasizing the similarities and differences 
observed between the large-scale structure of time series and their corresponding visibility networks.

\section{CHARACTERIZATION OF TIME SERIES}

To achieve a complete characterization of the time series in [6], a combined use of local and global geometrical properties is introduced. Consider a time series, $Y(t) \equiv\left\{y_{t_{1}}, y_{t_{2}}, \ldots, y_{t_{N}}\right\}$, representing one sample of an ensemble, $\left\{Y_{T}\right\}$, of a numerical or empirical process of size $T$. Global properties are associated with affine transformations. We focus on series with constant increments $t_{i+1}-t_{i}=\Delta$, and we consider affine transformations such as $\Delta^{\prime}=\lambda \Delta, y^{\prime}=\Delta^{\omega} y$. We can define a global exponent $\omega \rightarrow \alpha$ if there is an exponent that keeps invariant the statistical description of the series, $Y_{\lambda T}(\lambda t) \sim \lambda^{-\alpha} Y_{T}(t)$. Local properties are associated with changes in the internal variable, $\left\{t_{i}\right\}$, keeping constant the size of the series. As in the previous case, a local exponent $\alpha_{\text {loc }}$ can be defined if $Y_{T}(\lambda t) \sim \lambda^{-\alpha_{\mathrm{loc}}} Y_{T}(t)$. It is worth remarking that, except for the case of self-affine series where $\alpha_{\text {loc }}=\alpha$, a proper description of the scaling properties of time series requires at least two distinct exponents, accounting for their local and global character.

In practice, the global behavior is quantified by taking the scaling exponents of the asymptotic variation of moments with $T$ as the mean value $\left\langle\overline{Y_{T}}\right\rangle \sim T^{v}$ and variance $\left\langle\overline{\left(Y_{T}-\overline{Y_{T}}\right)^{2}}\right\rangle \sim$ $T^{2 \alpha}$. From now on, \# and $\langle \#\rangle$ denote time and sample averaging, respectively. To measure local properties, a wide variety of methods, ranging from spectral to wavelet analysis, have been used in the literature. As in the previous case, we are going to use methods that produce exponents to characterize the series. When the curve representing the series is self-affine, as in the case of $\mathrm{fBm}$ series, it is very frequent the use of the scaling of local widths as $\left.\overline{\left\langle[Y(t+\tau)-Y(t)]^{2}\right.}\right\rangle \sim \tau^{2 \alpha_{w}}$. The exponent $\alpha_{w}$ is also called the Hurst exponent. In the case of noises such as fBn, $\alpha_{w} \equiv 0$, and one can use as a quantifier the Hurst exponent of the accumulated series, which is an indirect measure. It is more convenient to use a direct measure as the correlation $C(t, T)=\langle\overline{Y(t) Y(T)}\rangle \sim t^{2 \phi}\left(\frac{T-t}{t}\right)^{2 \phi_{l}}[10]$ or the spectral density $S(f)=\frac{1}{T}\left\langle\widehat{Y_{T}(f)} \widehat{Y_{T}(-f)}\right\rangle \sim \frac{T^{2 \beta}}{f^{2 \alpha_{S}+1}}$ [11], where $\widehat{\#}$ is a Fourier transform. The advantage of using spectral densities is that they are well defined in all types of series. $\alpha_{s}$ is positive for fractal curves and negative for noises, whereas $\alpha_{w}$, which is always positive, is only defined for fractal curves, and $\phi_{l}>-1$. Moreover, the exponents in their common ranges are related to the others. In fractal curves, $\alpha_{s}$ and $\alpha_{w}$ coincide with the exception of the persistent fBm case with $\alpha_{w}>1 / 2$, where the spectral exponent saturates $\alpha_{s}=1 / 2$. Then a good characterization of the local scaling is by means of an exponent that we call $\alpha_{\text {loc }}$ defined as

$$
\alpha_{\mathrm{loc}} \equiv \begin{cases}\alpha_{s} & \text { if } \alpha_{s}<-1, \\ \alpha_{s}=\phi_{l} & \text { if }-1 \leqslant \alpha_{s} \leqslant 0 \\ \alpha_{s}=\phi_{l}=\alpha_{w} & \text { if } 0<\alpha_{s}<1 / 2 \\ \max \left\{\alpha_{s}=\phi_{l}, \alpha_{w}\right\} & \text { if } \alpha_{s} \geqslant 1 / 2\end{cases}
$$

Hence, if we restrict our attention to typical time series that are either symmetric with respect to their mean values $(v=0)$ or completely asymmetric $(v=\alpha)$, we can conclude, as in [6], that we have two independent exponents, $\alpha$ and $\alpha_{\text {loc }}$, which are suitable for a proper characterization of time series based on scaling properties. These exponents can be directly obtained from power spectra of series with variable size. In [12] it was shown that a complete scaling of power spectra can be written as

$$
S(f, T) \sim \begin{cases}\frac{T^{2\left(\alpha-\alpha_{s}\right)}}{f^{2 \alpha_{s}+1}} & \text { if } \alpha_{s} \geqslant 0, \alpha \neq 0, \\ \frac{[\log (T)]^{-1}}{f} & \text { if } \alpha_{s}=0, \alpha=0, \\ \frac{T^{2 \alpha}}{f^{2 \alpha_{s}+1}} & \text { if } \alpha_{s}<0 .\end{cases}
$$

Therefore, a correspondence of the distinct scaling exponents with the global exponent $\alpha$ can be written as

$$
\alpha \equiv \begin{cases}\beta & \text { if } \alpha_{s}<-1 / 2 \\ \beta=\phi-\phi_{l} & \text { if }-1 / 2 \leqslant \alpha_{s} \leqslant 0 \\ \beta+\alpha_{s}=\phi & \text { if } 0<\alpha_{s}\end{cases}
$$

The scaling of the spectra defines two general aspects of the large-scale structure of time series. On the one hand, there is an essential difference between noise $\left(\alpha_{s}<0\right)$ and fractal curves $\left(\alpha_{s}>0\right)$ that induces two different forms of scaling. On the other hand, one or two spectral scales exist in the dynamics of time series, which is another essential property. More specifically, with both exponents we can classify time series into classes, representing distinct types of series that share some geometrical property. This is very useful for our propose of comparing structures of series and visibility networks since every class of time series should map to their corresponding class of complex network. A diagram showing this classification and the representation of classes we are going to use in this paper is given in Fig. 1. We are going to consider five classes exhibiting distinct features:

(i) The class of self-affine curves (SA) defined by $\alpha_{\mathrm{loc}}=$ $\alpha>0$. A graphical representation of time series in this class is self-affine fractal curves since local and global scaling coincide. fBm curves are typical examples of this class. For their numerical implementation we use the Levinson

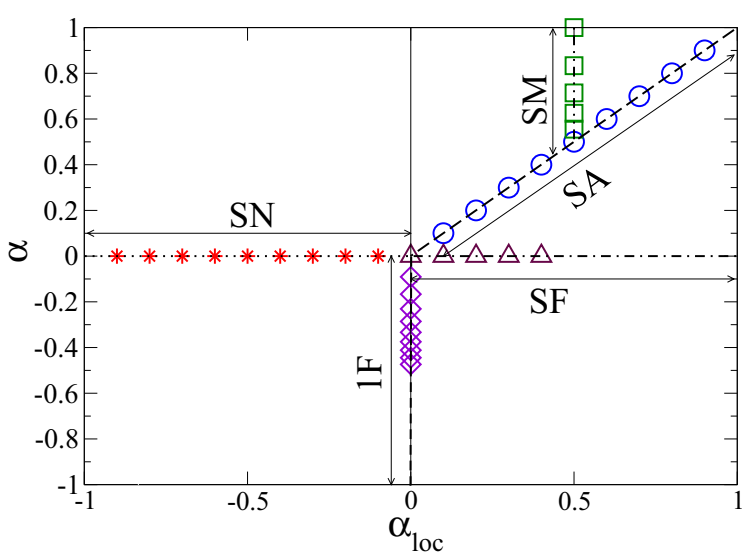

FIG. 1. Diagram of the classification of time series concerning their large-scale structure, using two exponents characterizing a local, $\alpha_{\text {loc }}$, and global, $\alpha$, behavior. Time series are ordered in classes: selfaffine (SA), stationary noise (SN), stationary fractal (SF), saturated multiplicative $(\mathrm{SM})$, and $1 / f(1 \mathrm{~F})$. Points in each class represent the cases under study. 
algorithm, which produces Gaussian fBm processes with a very accurate exponent $\alpha_{\text {loc }}=H$ [6].

(ii) The class of stationary noise (SN) defined by $\alpha_{\text {loc }}<0$, $\alpha=0$. It is composed of stationary correlated noise since its variance is constant with $T$ and the correlation is only dependent on the difference of times $\tau$. Series in this class can be numerically obtained using methods of pulse addition [6] or directly using $\mathrm{fBn}$ processes. In this paper, we use $\mathrm{fBn}$ series generated from increments of $\mathrm{fBm}$ processes which also gives Gaussian processes with $\alpha_{\text {loc }}(\mathrm{fBn})=\alpha_{\text {loc }}(\mathrm{fBm})-1$.

(iii) The class of saturated multiplicative processes (SM) defined by $\alpha_{\mathrm{loc}}=1 / 2, \alpha>1 / 2$. Multiplicative processes generated by the stochastic equation in the Ito interpretation:

$$
\dot{Y}(t)=(\mu-\rho) Y^{2 \mu-1}+\xi(t) Y(t)^{\mu},
$$

where $\xi(t)$ is a random Gaussian noise, present scaling exponents $[6,13]$ given by

$$
\begin{gathered}
\alpha_{\mathrm{loc}}=\frac{3-\rho}{4(1-\mu)}, \\
\alpha=\frac{1}{2(1-\mu)} .
\end{gathered}
$$

$\rho$ is the exponent of the probability density function, which in a certain region of $Y$ evolves as $P(Y, t) \sim Y^{-\rho}$. From (5) one obtains the condition of saturation of the local scaling, where $\alpha_{\text {loc }}=1 / 2$, as $\mu>\frac{\rho-1}{2}$. In these conditions, we have a process with an apparent form of random walk, since $\alpha_{\text {loc }}=$ $1 / 2$, that is not self-affine, $\alpha>\alpha_{\text {loc }}$. These are what we call SM processes. In our numerical implementation, we take powers of the Wiener process (fBm with $H=1 / 2) Y(t)=|W(t)|^{q}$, which lead to multiplicative processes with $\alpha_{s}=\frac{2 q+1}{4}$ and $\alpha=q / 2$. For $q>1 / 2, \alpha_{s}$ saturates to $1 / 2$ and series in the SM class are generated.

(iv) The class of $1 / f$ noise defined by $\alpha_{\text {loc }}=0, \alpha<0$. A standard generator of $1 / f$ noise consists of the addition of pulses whose interpulse time follows a positive randomwalk process [14]. Exponents of this process are $\alpha_{\mathrm{loc}}=0, \alpha=$ $-1 / 3$ [6]. Hence it is a good example of nonstationary noise with decreasing variance. A generalization of this process can be easily achieved simply by taking as the interpulse time process a positive fBm process with a given $\alpha_{\mathrm{loc}}=H$ [12]. Now the local scaling does not change, $\alpha_{\text {loc }}=0$, but the global scaling is dependent on $H$ as $\alpha=-\frac{H}{1+H}$. Hence a continuous band of $1 / f$ processes in the range $\alpha \in(0,1 / 2)$ can be obtained in this simple form. They are processes belonging to the $1 / f$ class. We have implemented them using a superposition of exponential pulses with amplitude unity and interpulse times given by the absolute value of $\mathrm{fBm}$ processes.

(v) The class of stationary fractal curves (SF) defined by $\alpha_{\text {loc }}>0, \alpha=0$. They are processes with a singular structure, since they are fractal curves $\left(\alpha_{\text {loc }}>0\right)$ with a constant variance $(\alpha=0)$, which appears in some physical experiments [15]. They are good examples of weak stationarity. They can be numerically implemented [6] by superposing pulses with random unitary amplitudes $( \pm 1)$ whose interpulse time process is a renewal process with a power-law distribution $P(\tau)=(d-1) \tau^{-d}$ with $1<d<2, \tau \in(1, \infty)$. The exponents obtained in this way are $\alpha=0, \alpha_{\text {loc }}=1-d / 2$ in a range $\alpha_{\text {loc }} \in(0,1 / 2)$.

\section{LARGE-SCALE STRUCTURE OF COMPLEX NETWORKS}

The most used characteristic to define a network structure is the distribution of vertex degrees $p_{k}$, defined as the fraction of vertices that have degree $k$ [7]. Depending on the asymptotic form of $p_{k}$, a first classification of networks is possible. We focus in this paper on the case in which this asymptotic form becomes a power law, $p_{k} \sim k^{-\gamma}$, and the network is then said to be scale-free. These kinds of networks are very common in many fields of science [7], usually with values $2 \leqslant \gamma \leqslant 3$, but our interest here is because they are typical of visibility networks.

There are some problems in detecting power-law behavior in a simple histogram such as $\left\{p_{k}\right\}$. One is that the histogram can be very noisy at the end of the tail due to statistical fluctuations. Then it is possible either to use a more suitable form of representation, or, if we have an ensemble of equivalent networks, consider sample-averaged histograms as $P_{k}=\left\langle p_{k}\right\rangle$. Typically, a real power-law distribution deviates from the true power law in the small- $k$ regime, but also this deviation can happen for high $k$ as well. There will be a cutoff of some type limiting high degrees of vertices in the tail. As we can see in the following sections, these limitations should be carefully treated in visibility networks to avoid misinterpretations.

The exponent of the power distribution $\gamma$ plays a similar fundamental role in complex networks to that $\alpha_{\text {loc }}$ in time series. In addition, as in the case of time series, we could say that in many situations it is not enough to define properly the large-scale structure of the network. If we deal with networks whose size is variable, or can be varied, it is possible to get a new characteristic by considering the scaling of the maximum degree with the size, $\left\langle k_{M}\right\rangle \sim N^{\kappa}$. In visibility networks, it is expected that this exponent $\kappa$ plays the same role as the global exponent $\alpha$ in their corresponding time series.

The physical structure of networks imposes constraints on the value of the characteristic exponents $\gamma$ and $\kappa$. There is a general constraint that is called the natural bound [16], $\kappa \leqslant \frac{1}{\gamma-1}$, which is not due to structural constraints but is inherent in any finite degree sequence with a power-law distribution. When the networks are standard, with neither multiple connections nor self-connections, a typical structural constraint comes from the conditions of graphicality. For generic scale-free networks, this condition reads $\kappa<1 / \gamma$ in the range $1<\gamma<2$. The constraints imposed by graphical conditions in visibility networks have been treated in [17]. It is worth remarking that these constraints apply to networks whose degree distributions exhibit true power laws without size dependence. As we will see in the following sections, degree distributions of visibility networks mapped from double scaling series are size-dependent, and new constraint rules should be adapted for them.

\section{TIME SERIES AND VISIBILITY NETWORKS}

Visibility graphs from time series are produced by considering that two data points that are mutually visible are connected. In more concrete terms, any pair of data points $y_{t_{i}}, y_{t_{j}}\left(t_{i}<t_{j}\right)$ are mutually visible, and hence they are connected in the corresponding graph, if and only if for any intermediate 
$t_{k}, t_{i}<t_{k}<t_{j}$

$$
y_{t_{k}}<y_{t_{i}}+\left(y_{t_{j}}-y_{t_{i}}\right) \frac{t_{k}-t_{i}}{t_{j}-t_{i}} .
$$

Note that by construction the visibility graph is undirected and always connected, since each node is linked at least with its neighbors. One important property is the invariance of the visibility graph under affine transformation of the series, thus affine transformation in time series is irrelevant in their corresponding visibility graph, which indicates that they capture only a part of the structure. A given visibility network represents not only the corresponding series but also all series produced by affine transformations. But this also means that not all transformations done on time series can be detected by visibility networks. In fact, there is not a direct relationship between the classification with exponents $\alpha$ and $\alpha_{\text {loc }}$, based on correlations and spectral densities, and the classification of visibility networks based on exponents $\gamma$ and $\kappa$. While in the first case the exponents account for independent properties, this is not so clear in the second. For instance, we only need the exponents $\alpha_{\text {loc }}$ and $\alpha$ to classify almost every kind of time series, whereas, as we will see, a third exponent added to $\gamma$ and $\kappa$ is necessary to represent the same sets. This happens because degree distributions of visibility networks coming from double-scaling time series are size-dependent, $P_{k} \sim N^{\theta} k^{-\gamma}$, and in general there is not a direct dependence, such as the Parcival law in spectral analysis [6], between the new exponent $\theta$ and $\kappa$. Note that with this scaling of the degree distribution, the natural boundary condition changes. Since it is given by the condition $N \int_{k_{M}}^{\infty} P_{k} d k<1$, now it reads

$$
\kappa<\frac{1+\theta}{\gamma-1} \text {. }
$$

\section{NUMERICAL RESULTS AND THEIR INTERPRETATION}

In the degree distribution of scale-free networks, the first and final part of the power-law distribution can deviate appreciably from their theoretical distribution, it being difficult, in some cases, to get the correct behavior with only samples of one size. Deviations in the first part of the distribution are usually due to finite-size effects, but deviations of the last part can be due to true structures that scale with the size of the network producing characteristic patterns in the distribution. On the other hand, as we have mentioned, series with double scaling in their spectral density produce networks with size-dependent degree distributions of the form $P_{k} \sim N^{\theta} k^{-\gamma}$. Hence, an analysis with series of distinct sizes is necessary to capture the true large-scale structure of both the time series and their corresponding visibility networks. To obtain the scaling pattern of visibility networks, we use the averaged histogram of the relative number of edges with respect to the maximum value, $P_{k / k_{M}}=\left\langle p_{k / k_{M}}\right\rangle$, for distinct sizes. If the distribution is a size-dependent power law with exponents $\gamma$ and $\theta$, this quantity scales as $\left\langle k_{M}\right\rangle^{\eta} P_{k / k_{M}} \sim\left(k / k_{M}\right)^{-\gamma}$, with $\eta=\gamma-1-\theta / \kappa$. We plot in log-log the left part of this equivalence against $k / k_{M}$ for distinct sizes, varying $\eta$ until reaching a collapse of the curves as in Figs. 6, 7, and 8. The power law exists and the exponent is correct if, besides a good collapse, the slope of the collapsed part is the exponent $\gamma$. In this way, the pattern of the distribution composed of a true power law and deviations is perfectly identified. Finally, the exponent $\kappa$ is obtained straightforwardly by plotting in a $\log$-log representation $\left\langle k_{M}\right\rangle$ against $N$ for different sizes. In the analysis that follows, we have used time series with sizes $2^{12}$, $2^{14}$, and $2^{16}$ and ensembles of 100 samples. In some cases, the finite-size effects have a slow variation, and very long series are needed to get the true exponents. A recent fast algorithm [18] to create visibility graphs from time series allows us to deal with series up to $2^{20}$ points in a reasonable amount of computing time.

\section{A. Time series with one spectral scale: SA and SN classes}

As mentioned in Sec. II, we use Gaussian fBm processes to represent the SA class and time increments (also Gaussian) of them to represent the SN class. The SA class consists of time series with a self-affine graphical representation in which the local and global scaling coincide, $\alpha_{\text {loc }}=\alpha=H>0$. It is worth noting that a small difference in structure exists between the antipersistent and the persistent series. As we are going to see, this difference also affects the structure of their corresponding visibility networks. So, regarding the structure of time series, we will consider three cases in this subsection composed of series in the SA class with (i) persistent behavior $\left(\alpha=\alpha_{\mathrm{loc}}=H \geqslant 0.5\right)$, (ii) antipersistent behavior $\left(\alpha=\alpha_{\text {loc }}=\alpha_{s}=H<0.5\right)$, and (iii) series in the complete SN class $\left(\alpha_{\mathrm{loc}}=\alpha_{s}<0, \alpha=0\right)$. To represent these cases, we use, respectively, fBm processes with $H=0.7$, $H=0.3$, and $\mathrm{fBn}$ processes with $H=0.5$.

On the one hand, let us consider the scaling of the maximum degree with the system size through the exponent $\kappa$ as $\left\langle k_{M}\right\rangle \sim N^{\kappa}$. As mentioned in [17], $\kappa$ is in fact the fractal dimension of the visible sites from the site of maximum visibility. Given the self-affine property of the series in the SA class, a close connection between the exponents $\alpha=\alpha_{\text {loc }}$ and this fractal dimension can be expected. In fact, the identity $\kappa=\alpha=H$ is supported by the numerical analysis shown in Fig. 2. Differences observed in the extremes $H \rightarrow 1$ and $H \rightarrow 0$ are due to finite-size effects. These finite-size effects are very important in the entire class of SN series. As shown in Fig. 2, an estimated value of $\kappa \sim 0.2$ is obtained with a standard analysis, but when using a more detailed description to see finite-size effects, an extremely slow variation, $\kappa \sim$ $1 / \log (N)$, is observed, confirming that in this class $\kappa=0$ is the true exponent. Hence, the identity $\kappa=\alpha$ is numerically corroborated.

On the other hand, the analysis of the degree distribution is more complicated and requires a careful study of the scaling with the system size $N$. In Fig. 3, this analysis is outlined. Typical degree distributions of visibility graphs are depicted in the left panels for the representatives of each case. One can see that the three cases possess very distinct structure. The dashed line, which corresponds to the curve $\exp \left(-k^{\mu_{1}}\right)$, with $\mu_{1} \sim 0.5$, fits perfectly in the entire range of degrees in the $\mathrm{SN}$ case, whereas it fits only the zone of small degrees in the other cases. In the central panels of Fig. 3, we plot the scaled distribution of relative degrees $\left\langle k_{M}\right\rangle^{\gamma^{*}-1} P\left(k / k_{M}\right)$ against $k / k_{M}$, adjusting $\gamma^{*}$ to get a collapse of the curves with distinct $N$. If a true power law exists, the exponent $\gamma^{*}$ 

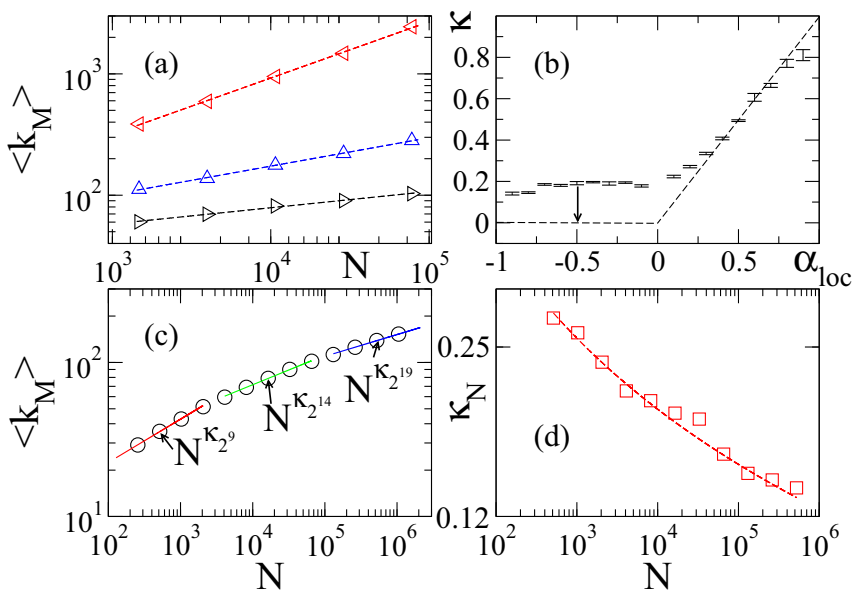

FIG. 2. (a) Estimation of the exponent $\kappa$ from the scaling $\left\langle k_{M}\right\rangle \sim$ $N^{\kappa}$ for visibility networks corresponding to fBm series with $\alpha_{\mathrm{loc}}=$ $H=0.7$ (persistent, left triangle), $\alpha_{\mathrm{loc}}=H=0.3$ (antipersistent, up triangle), and $\mathrm{fBn}$ series with $\alpha_{\mathrm{loc}}=\alpha_{s}=-0.5$ (white noise, right triangle). Values are obtained from an average of the maximum degree in 100 samples and series sizes of $2^{8}, 2^{10}, 2^{12}, 2^{14}, 2^{16}$. (b) Values of $\kappa$ obtained with this method for the entire range $\alpha_{\text {loc }} \in(-1,1)$ including series in the SN and SA classes. The dashed line corresponds to $\kappa=\alpha$. (c) More precise estimation of $\kappa$ for a series in the SN class $\left(\alpha_{s}=-0.5\right)$. Now the number of samples is 1000 and the range of sizes goes from $2^{8}$ to $2^{20}$. A small curvature is observed. (d) Local exponents $\kappa_{N}$ vs $N$ obtained in the left-hand figure. The dashed red line is the fitted curve, $\kappa_{N}=\frac{1.79}{\log N}$.

found in the collapse should be coincident with the exponent of the observed power law in the figure $\left(k / k_{M}\right)^{\gamma^{*}}$. This clearly happens in the persistent case (first row), where a
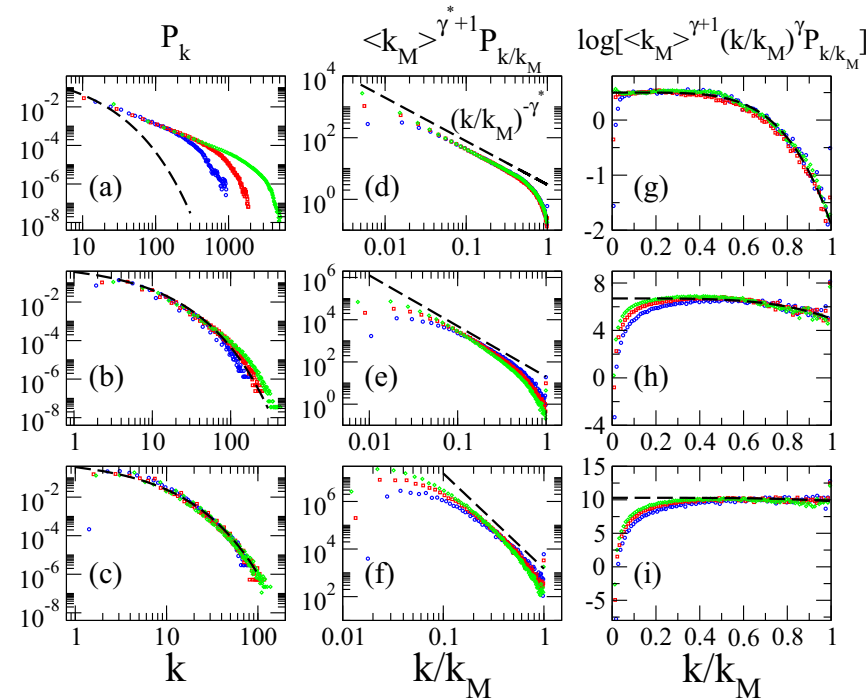

FIG. 3. Three distinct scaled plots of the degree distribution to account for behaviors of the smallest degrees, $P_{k} \sim \exp -a k^{\mu_{1}}[(\mathrm{a})-$ (c)]; intermediate ones, $P_{k / k_{M}} \sim\left(k / k_{M}\right)^{-\gamma^{*}}$ [(d)-(f)]; and highest degrees, $P_{k / k_{M}} \sim\left(k / k_{M}\right)^{-\gamma} \exp \left[-A\left(k / k_{M}\right)^{\mu_{2}}\right][(\mathrm{g})-(\mathrm{i})]$, for the same three series used in the previous figure: $\alpha_{\mathrm{loc}}=-0.5$ [(c), (f), and (i)], $\alpha_{\mathrm{loc}}=0.3$ [(b), (e), and (h)], and $\alpha_{\mathrm{loc}}=0.7$ [(a), (d), and (g)]. Dashed lines correspond to the fitted curves. perfect pattern of the distribution is observed: a first part with deviations due to finite-size effects, a second part that is a true power law (now the exponent is true, $\gamma=\gamma^{*}$ ), and a final part with an exponential form that is size-dependent. So, a more complete scaling as $\sim\left(k / k_{M}\right)^{\gamma} \exp \left[-A\left(k / k_{M}\right)^{\mu_{2}}\right]$ is expected in this case. In the other two cases, the curves in the intermediate zone of degrees only collapse at one point, indicating that $\gamma^{*}$ is not a true exponent. These exponents have been taken as true in other works in which simple analyses with only one size were performed [2]. These apparent exponents obtained in the entire range of SA and SN classes are given by a simple law, $\gamma^{*}=3-2 \alpha_{\text {loc }}$. Moreover, these exponents have been justified through some scaling arguments [5] that assume the existence of true power laws, but in [19] doubts about the validity of their numerical estimation are expressed.

To obtain the new exponent $\mu_{2}$, as shown in the righthand panels of Fig. 3, a plot of $\log \left[\left\langle k_{M}\right\rangle^{\gamma+1}\left(k / k_{M}\right)^{\gamma} P\left(k / k_{M}\right)\right]$ versus $k / k_{M}$, adjusting the exponent $\gamma$ to reach a collapse of the zone with the highest degrees, is performed. The fit with the expected curve $\log B-A x^{\mu_{2}}$ is complete in the case of persistent SA series. The case of antipersistent $\mathrm{fBm}$ series is not so clear since, although the collapse exists, the separation between the exponential and power-law parts, implying the existence of a flat slope, is limited by finite-size effects that are stronger since the range of scaling is smaller $\left(\sim N^{\kappa}\right)$.

A plot of the exponents obtained in the complete range of SA and SN processes is shown in Fig. 4, where a distinction between true and apparent exponents is given. In this interpretation, it is assumed that the true behavior of the networks from SN processes is $\exp \left(-a k^{\mu_{1}}\right)$, so the other exponents $\gamma$ and $\gamma^{*}$ are only apparent. For SA processes, we assume a true scaling, $\left(k / k_{M}\right)^{\gamma} \exp \left[-A\left(k / k_{M}\right)^{\mu_{2}}\right]$, with $\gamma=1 / \kappa$, so the exponents $\gamma^{*}$ are true in persistent models, where $\gamma=\gamma^{*}$, but only apparent in antipersistent models. Note the great difference between these exponents and the ones obtained in previous work, where $\gamma=3-2 \alpha_{\text {loc }}$ [2]. We have, in fact, a simple heuristic argument that supports the exponent

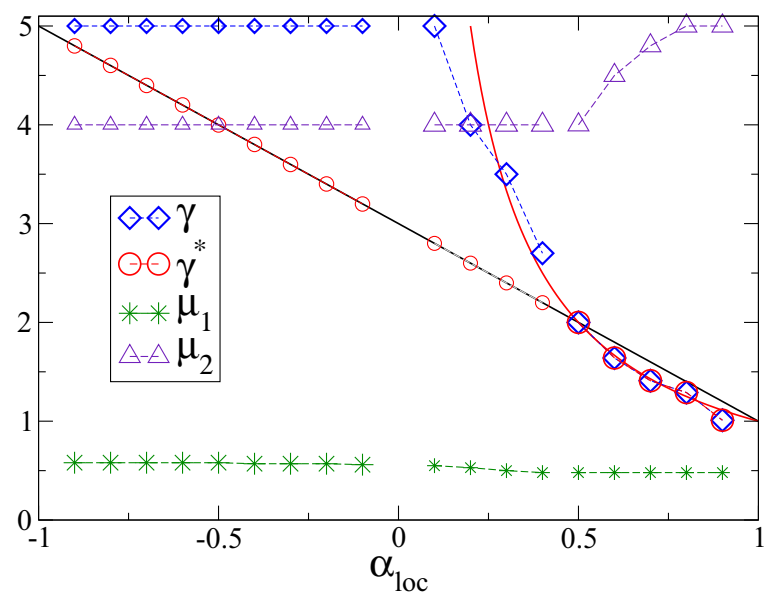

FIG. 4. Exponents obtained from the three scaled plots of the previous figure for the entire range $\alpha_{\mathrm{loc}} \in(-1,1)$. The larger symbols correspond to true exponents, and the smaller symbols correspond to apparent exponents. Continuous lines represent the models: red, $\gamma=1 / \kappa=1 / \alpha_{\mathrm{loc}} ;$ black, $\gamma=3-2 \alpha_{\mathrm{loc}}$. 
$\gamma=1 / \kappa$ in visibility networks coming from SA series. This argument uses the idea of separation in isolated blocks that yields such good results in simulations [18]. Given a series of size $N$, the highest point separates the support of the series into two parts whose points are not mutually visible. Assuming that this is the point of maximum visibility with a number of edges proportional to $N^{\kappa}$, and that the separated blocks have a similar number of points $N_{1} \sim N / 2$, one sees that in the $i$ th partition we have $2^{i}$ points with maximum visibility in zones with lengths $\frac{N}{2^{i}}$, that is, with $\left(\frac{N}{2^{i}}\right)^{\kappa}$ edges. Then one obtains the scaling $p_{k} \sim k^{-1 / \kappa}$, that is, $\gamma=1 / \kappa$. In simple simulations, one sees that if partitions are not of the same size but random, the scaling keeps its shape. So the exponential cutoff observed at the maximum edges should be a border effect.

\section{B. Time series with two spectral scales: SM 1F and SF classes}

One interesting result presented in this paper concerns the size dependence of the degree distribution of a visibility network when the corresponding time series has a spectral power depending on size. As an illustrative example, we show in Fig. 5 a case in the SM class with $\alpha=1$ and $\alpha_{\text {loc }}=0.5$. The size dependence of the spectra $S(f, T) \sim T f^{-2}$ translates into size dependence of the degree distribution $P_{k} \sim N^{0.35} k^{-2.6}$. Hence, time series with two spectral scales map, in general, to visibility networks whose degree distributions are also size-dependent. Note that if the degree density is given by a pure power law with exponent $-\gamma$ in the interval $\left(k_{m}, k_{M}\right)$, with $k_{M} \gg k_{m}$ and $\gamma>1$, we have

$$
P_{k}=\frac{\gamma-1}{k_{m}^{1-\gamma}-k_{M}^{1-\gamma}} k^{-\gamma} \sim(\gamma-1) k_{m}^{\gamma-1} k^{-\gamma},
$$

which implies a size dependence of the minimum degree as $k_{m} \sim N^{\frac{\theta}{\gamma-1}}$. This is the case observed in Fig. 5. In a more general case with the existence of crossovers as in Fig. 6, the
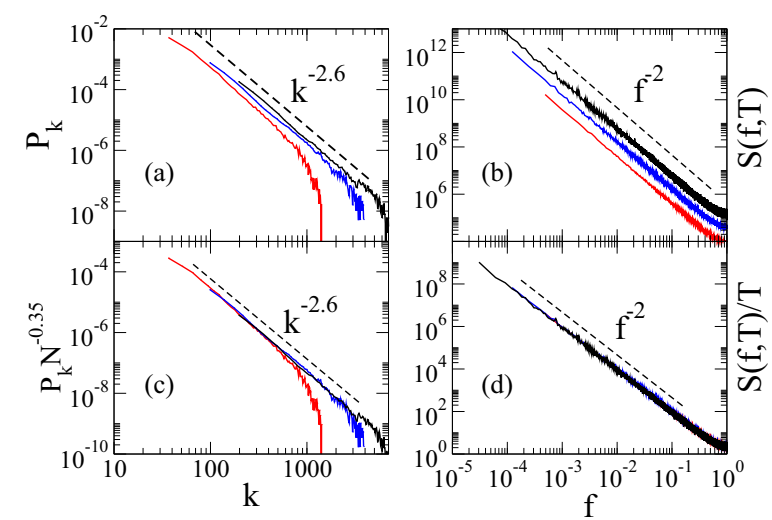

FIG. 5. Series in classes with double spectral scaling, whose spectra are size-dependent, produce visibility networks whose degree distributions are also size-dependent. Right: PSD's for series with three sizes $2^{12}, 2^{14}, 2^{16}$ in their normal form (b) and collapsed (d). Left: degree densities of the corresponding visibility networks in their normal form (a) and collapsed (c). Series belong to the multiplicative class with $\alpha_{s}=0.5, \alpha=1$. The measured exponents of their corresponding visibility networks are $\theta=0.35$ and $\gamma=2.6$. Spectra and degree densities are averaged using 100 samples.

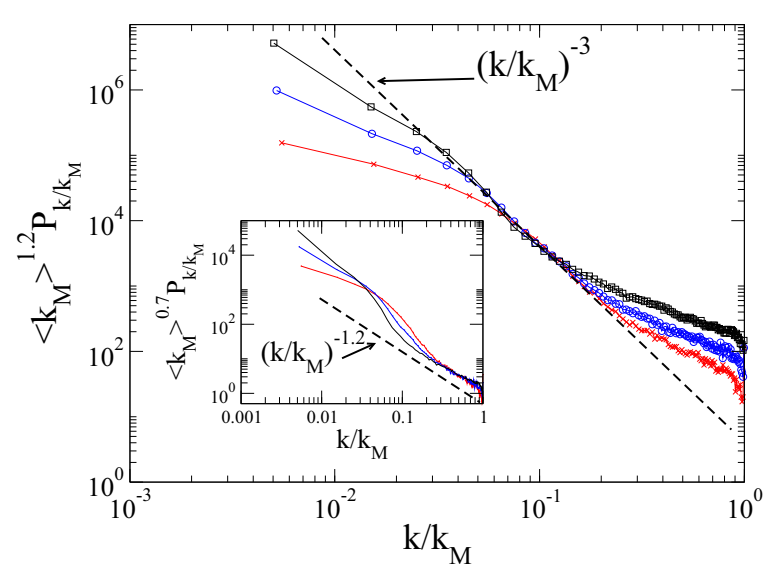

FIG. 6. Scaled relative degree densities of visibility networks corresponding to a multiplicative series with $\alpha_{s}=0.5, \alpha=1.5$ showing a crossover between two states with power laws. The exponential behavior observed in the part with the highest degrees of the previous cases is substituted here by a power law.

size dependence of the intervals where the power laws are observed is more complicated.

SM class. Numerical simulations indicate that the three exponents $\kappa, \gamma$, and $\theta$ are connected through $\eta=\gamma-1-$ $\theta / \kappa \sim 1$ in visibility networks coming from processes in the SM class. Using (7) one sees that a natural bound does not exist in this case, since it reads $\kappa<1$. However, when passing the standard natural bound, for $\alpha>1$, a kind of crossover as seen in Fig. 6 is observed in the relative degree density. Instead of having an exponential cutoff, as in the precedent cases, we observe a kind of power law. So it is clear that a transition in the behavior of multiplicative processes for $\alpha>1$ is being detected, and this change is not so clearly observed with standard methods of time series analysis.

$1 F$ class. Visibility networks of processes in the $1 \mathrm{~F}$ class also present a two-scale behavior as shown in Fig. 7. One interesting question that arises in this case is whether the

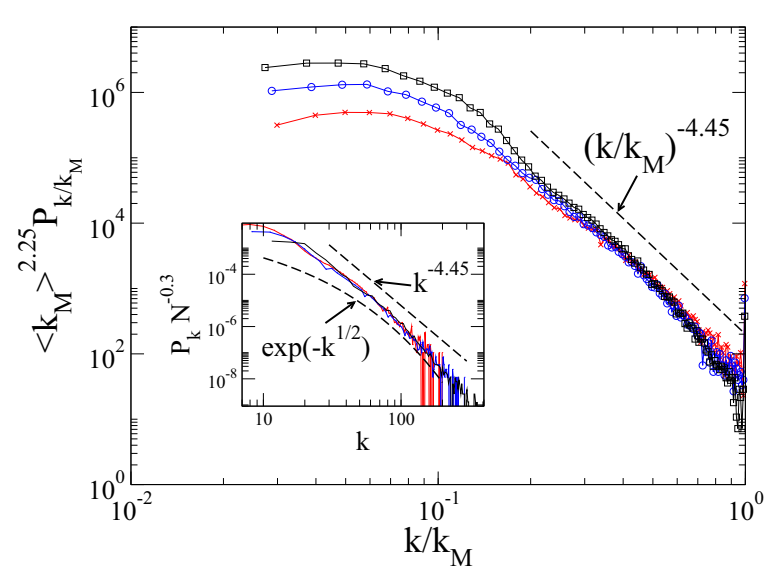

FIG. 7. Scaled relative degree densities of visibility networks corresponding to a series in the $1 \mathrm{~F}$ class $\left(\alpha_{s}=0, \alpha=-0.3\right)$. In the inset, the explicit dependence with $N$ is shown. The dashed lines are guides for the eye to compare true power-law and exponential behavior. 


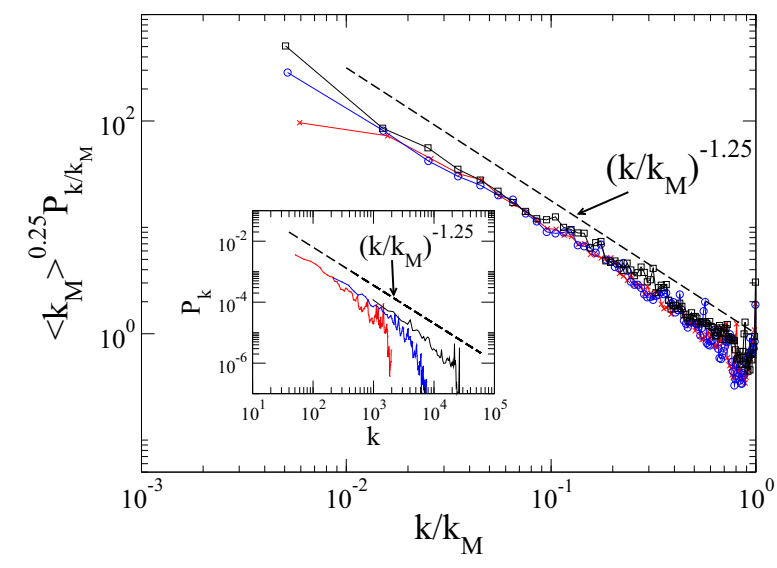

FIG. 8. Scaled relative degree densities of visibility networks corresponding to a series in the SF class $\left(\alpha=0, \alpha_{s}=0.3\right)$. In the inset, the lack of dependence with the series size is shown.

degree distribution exhibits a true power law or if it is simply exponential as in the SN class. In the inset of the figure, both alternatives are confronted. Although the possibility of a power law seems more evident, the small range of scales involved in the analysis precludes a definitive answer. In fact, for $-\alpha>0.3$ numerical results seem to overcome the corresponding natural barrier, which is an indication that a power law is not possible. In this case, one obtains $\kappa \sim \theta$, so, from (7), the natural bound reads $\kappa<\frac{1}{\gamma-2}$. As seen in Fig. 9, this bound is overcome.

$S F$ class. Visibility networks mapped from processes in the SF class show an extreme behavior with $\gamma \sim 1$. Despite the double scaling observed in the spectral densities [6], the degree density of the corresponding visibility networks does not present any apparent size dependence (Figs. 8 and 9). Scale-free networks with an exponent less than 2 show different properties [20]. Probably the broad dispersion of
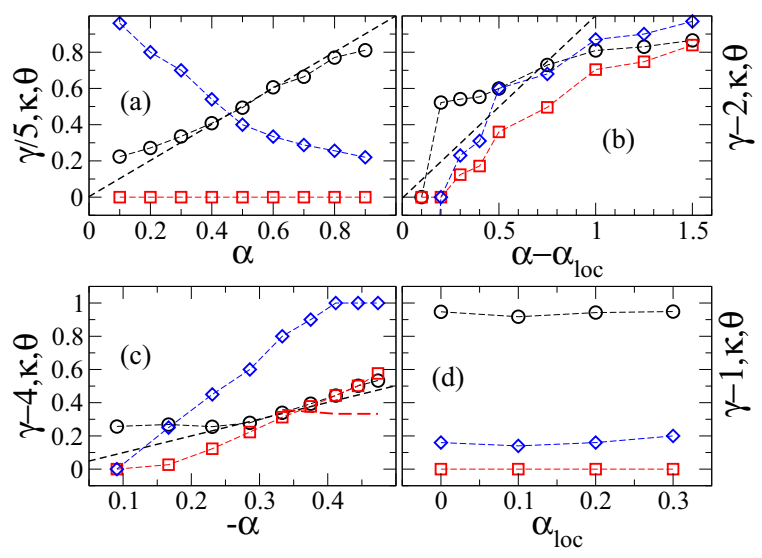

FIG. 9. Exponents $\gamma, \kappa$, and $\theta$ obtained from visibility networks corresponding to series in the SA (a), SM (b), $1 \mathrm{~F}(\mathrm{c})$, and SF (d) classes in a wide range of cases. Exponents $\gamma$ and $\theta$ are obtained from the scaling of the degree distribution, $P_{k} \sim N^{\theta} k^{-\gamma}$, as in Fig. 5. Exponent $\kappa$ is obtained from the scaling of the mean maximum degree, $\left\langle k_{M}\right\rangle \sim$ $N^{\kappa}$, as in Fig. 2(a). Black dashed lines are $y=x$ lines to guide the eye. The red dashed line in (c) is the natural barrier calculated from (7). degrees in the local behavior facilitates the lack of dispersion in the global one.

Finally in Fig. 9, and for the sake of completeness, a plot of exponents $\gamma, \kappa$, and $\theta$ versus the relevant spectral exponents is shown for series in the SA, SM, 1F, and SF classes.

\section{CONCLUSIONS}

In this paper, we have dealt with time series ordered in five classes whose power spectra exhibit clear power laws in practically the complete range $\sim T$ (Fig. 5), whereas the degree densities of their corresponding visibility networks present a structural cutoff, strong finite-size effects, and narrower ranges, $\sim T^{\kappa}$. So, from a technical point of view, the direct analysis of time series based on correlations or spectral densities is more feasible than the analysis of visibility networks. In fact, in this last case a detailed analysis involving size-dependent scaling is necessary to obtain true exponents. In this way, we have obtained different values of exponents than the ones shown in previous work. Therefore, I strongly recommend this type of detailed analysis in future uses of visibility networks to characterize either time series or other surfaces.

In Fig. 10, we present the counterpart in network theory of the time series diagram presented in Fig. 1. Now the large-scale structure of visibility networks is analyzed by means of $\gamma$ and $\kappa$, which play the role of exponents in a local and global scaling, respectively. Symbols in the diagram are numerical results of visibility networks mapped from the same time series as those used in Fig. 1, whose detailed description was given in the preceding section. Larger symbols correspond to true exponents, and smaller symbols to apparent ones. Continuous lines are theoretical values of single-scaled processes. Black represents the model of [5], $\gamma=3-2 \alpha_{\text {loc }}$, while red represents our model, $\gamma=1 / \kappa$, both with $\kappa=\alpha$. Comparing the diagrams, at first glance we see that networks present strong system-size effects, much more intense than

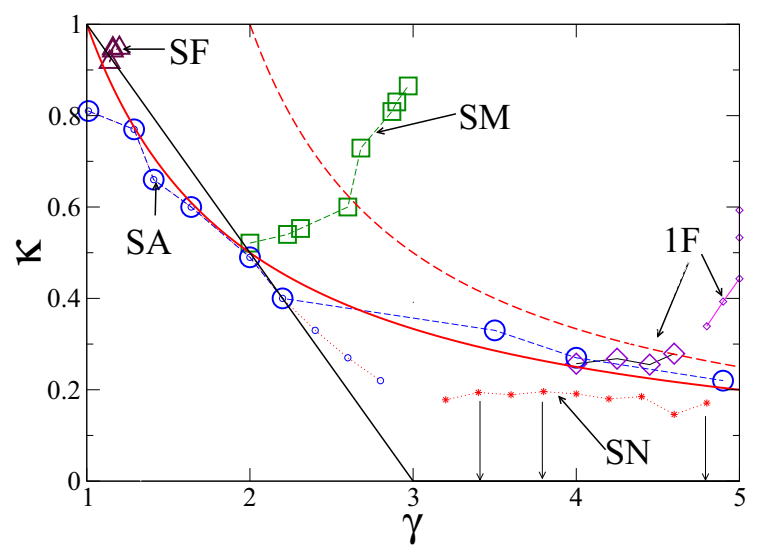

FIG. 10. Diagram of the classification of visibility networks concerning their large-scale structure, using two exponents characterizing a local, $\gamma$, and global, $\kappa$, behavior. Points in the diagram correspond to visibility networks mapped from the time series appearing in Fig. 1. The larger symbols represent true exponents, whereas the smaller symbols represent apparent exponents. The red dashed line is the natural barrier, and continuous lines represent models. 
their corresponding time series. We also observe that visibility networks can overcome the reference barriers of the standard networks. These facts were explained in the preceding section. Now we focus on the possible connection between the structures of time series and visibility networks. We can see that there are evident similarities but also deep differences, which implies that the analysis of visibility networks can provide us with information other than that obtained by correlations of time series. The main facts observed with correlations, or more specifically with spectral analysis, have an analog in the analysis of visibility networks. On the one hand, the clear difference between noise $\left(\alpha_{\text {loc }}<0\right)$ and fractal curves $\left(\alpha_{s}>0\right)$, observed, for instance, in the scaling of spectra, is transformed here into differences in the scaling laws of the degree distribution $P_{k}$. Visibility networks of fractal curves exhibit a true power-law distribution with an exponent $\gamma=1 / \kappa$, while degree distributions of noises are not power laws, since the exponent $\gamma^{*}$ obtained in other works is only an apparent exponent. On the other hand, single-scaled spectral processes observed in time series, which correspond to SA and $\mathrm{SN}$ classes, are mapped into visibility networks whose degree distribution is also single-scaled, that is, it is independent of the size as in any standard distribution. Double-scaled processes corresponding to the remaining classes map into networks with degree distributions that scale with the system size as $P_{k} \sim$ $N^{\theta} k^{-\gamma}$. So they are not standard power-law distributions, which implies that the usual restrictions of barriers, which come from asymptotic behavior, do not apply in these cases.
Furthermore, I consider that this behavior is not exclusive to visibility networks. Since it seems essential to describe the structure of growing networks, it may be interesting to investigate its existence in other types of networks.

Concerning differences, in the time series diagram of Fig. 1 one can see that the stationary $1 / f$ process $\left(\alpha_{\text {loc }}=0, \alpha=0\right)$ is a kind of critical point in which processes of four distinct classes converge. Close to this point, correlations are very similar despite being very different processes. In the diagram of networks, this point, which would correspond to $\gamma=3$, $\kappa=0$, does not exist. In fact, it is just the contrary, i.e., their corresponding processes appear very dispersed: $\kappa=0$, $\gamma=\infty$ in the SA class, $\kappa \sim 1, \gamma \sim 1$ in the SF class, and $\kappa=0, \gamma=0$ (not power laws) in the $\mathrm{SN}$ and $1 / \mathrm{F}$ classes. This dispersion suggests that the use of visibility networks to distinguish between types of $1 / f$ processes could be very efficient.

To end with two general conclusions, we can say that visibility networks are sensitive to different aspects of the structure of time series due to correlations, which means that they can be used as a complementary analysis of time series. Secondly, visibility networks are examples of growth networks with new kinds of scaling.

\section{ACKNOWLEDGMENT}

I acknowledge support from MINECO (Spain) under Projects No. Fis2014-59462-P and No. CGL2015-66583-R.
[1] J. Zhang and M. Small, Phys. Rev. Lett. 96, 238701 (2006).

[2] L. Lacasa, B. Luque, F. Ballesteros, J. Luque, and J. C. Nuño, Proc. Natl. Acad. Sci. (USA) 105, 4972 (2008).

[3] Q. Tang, J. Liu, and H. Liu, Mod. Phys. Lett. B 24, 1541 (2010).

[4] L. Telesca, M. Lovallo, A. Ramirez-Rojas, and L. FloresMarquez, Physica A 392, 6571 (2013).

[5] L. Lacasa, B. Luque, J. Luque, and J. C. Nuño, Europhys. Lett. 86, 30001 (2009).

[6] M. A. Rodríguez, Phys. Rev. E 90, 042122 (2014).

[7] M. E. J. Newman, Networks, An Introduction (Oxford University Press, Oxford, 2013).

[8] L. F. Costa, F. A. Rodrigues, G. Travieso, and P. R. Villas Boas, Adv. Phys. 56, 167 (2007).

[9] M. Boguñá, R. Pastor-Satorras, and A. Vespignani, Eur. Phys. J. B 38, 205 (2004).

[10] N. Leibovich and E. Barkai, Phys. Rev. Lett. 115, 080602 (2015).
[11] M. B. Priestley, Spectral Analysis and Time Series (Academic Press, San Diego, CA, 1996).

[12] M. A. Rodríguez, Phys. Rev. E 92, 012112 (2015).

[13] B. Kaulakys and J. Ruseckas, Phys. Rev. E 70, 020101(R) (2004).

[14] B. Kaulakys and T. Meskauskas, Phys. Rev. E 58, 7013 (1998).

[15] M. Niemann, H. Kantz, and E. Barkai, Phys. Rev. Lett. 110, 140603 (2013).

[16] R. Cohen, K. Erez, D. ben-Avraham, and S. Havlin, Phys. Rev. Lett. 85, 4626 (2000).

[17] M. A. Rodríguez, Phys. Rev. E 94, 012314 (2016).

[18] X. Lan, H. Mo, S. Chen, Q. Liu, and Y. Deng, Chaos 25, 083105 (2015).

[19] P. Manshour, Chaos 25, 103105 (2015).

[20] H. Seyed-allaei, G. Bianconi, and M. Marsili, Phys. Rev. E 73, 046113 (2006). 